Review Article

\title{
Revisiting Epithelial-to-Mesenchymal Transition in Liver Fibrosis: Clues for a Better Understanding of the "Reactive" Biliary Epithelial Phenotype
}

\author{
Luca Fabris, ${ }^{1,2}$ Simone Brivio, ${ }^{3}$ Massimiliano Cadamuro, ${ }^{1,3}$ and Mario Strazzabosco ${ }^{2,3}$ \\ ${ }^{1}$ Department of Molecular Medicine, University of Padua School of Medicine, Viale G. Colombo 3, 35131 Padua, Italy \\ ${ }^{2}$ Liver Center, Section of Digestive Diseases, Yale University, TAC Building, 333 Cedar Street, New Haven, CT 06520, USA \\ ${ }^{3}$ School of Medicine and Surgery, University of Milan-Bicocca, Via Cadore 48, 20900 Monza, Italy
}

Correspondence should be addressed to Massimiliano Cadamuro; massimiliano.cadamuro@unimib.it

Received 28 August 2015; Accepted 20 October 2015

Academic Editor: Pura Muñoz-Cánoves

Copyright (C) 2016 Luca Fabris et al. This is an open access article distributed under the Creative Commons Attribution License, which permits unrestricted use, distribution, and reproduction in any medium, provided the original work is properly cited.

\begin{abstract}
Whether liver epithelial cells contribute to the development of hepatic scarring by undergoing epithelial-to-mesenchymal transition (EMT) is a controversial issue. Herein, we revisit the concept of EMT in cholangiopathies, a group of severe hepatic disorders primarily targeting the bile duct epithelial cell (cholangiocyte), leading to progressive portal fibrosis, the main determinant of liver disease progression. Unfortunately, therapies able to halt this process are currently lacking. In cholangiopathies, fibrogenesis is part of ductular reaction, a reparative complex involving epithelial, mesenchymal, and inflammatory cells. Ductular reactive cells (DRC) are cholangiocytes derived from the activation of the hepatic progenitor cell compartment. These cells are arranged into irregular strings and express a "reactive" phenotype, which enables them to extensively crosstalk with the other components of ductular reaction. We will first discuss EMT in liver morphogenesis and then highlight how some of these developmental programs are partly reactivated in DRC. Evidence for "bona fide" EMT changes in cholangiocytes is lacking, but expression of some mesenchymal markers represents a fundamental repair mechanism in response to chronic biliary damage with potential harmful fibrogenetic effects. Understanding microenvironmental cues and signaling perturbations promoting these changes in DRC may help to identify potential targets for new antifibrotic therapies in cholangiopathies.
\end{abstract}

\section{Introduction}

Epithelial-to-mesenchymal transition (EMT) is a process of cellular reprogramming through which differentiated epithelial cells lose their native identity and acquire morphological and functional properties of mesenchymal cells, including a spindle-shaped ("fibroblast-like") appearance and the ability to detach from and to migrate outside the epithelial layer [1]. This process is relevant in physiological conditions, as seen during embryonic development, but it may occur also in pathological conditions, leading to organ fibrosis and malignant transformation in several organs [2].

The loss of epithelial cell-cell adhesion, caused by the relocalization and/or degradation of critical junction proteins, including E-cadherin, $\beta$-catenin, zonula occludens-1, occludin, and claudin, usually represents the first step of
EMT. E-cadherin loss is often counterbalanced by the aberrant de novo expression of $\mathrm{N}$-cadherin, an adhesion molecule enabling epithelial cells to establish dynamic interactions with surrounding mesenchymal cells. The disassembly of cell junctions, together with the ability to erode the basement membrane, results in a reduced intercellular cohesion within the epithelial layer [3, 4]. However, cells undergoing EMT also show a rearrangement of the cytoskeletal architecture, deriving from the downregulation of cytokeratins $(\mathrm{K})$ along with the upregulation of cytoskeletal proteins belonging to the mesenchymal lineage, including vimentin, S100A4 (also called fibroblast specific protein-1), and, eventually, $\alpha$-smooth muscle actin ( $\alpha$-SMA). These cytoskeletal and cell surface remodeling lead to the loss of the apical-basal polarity, typical of the epithelial phenotype, in favor of a front-rear polarity, prerequisite for the increased motility displayed by 
mesenchymal cells. Additional EMT changes include the ability to produce extracellular matrix (ECM) components, such as fibrillar collagen, fibronectin, elastin, and tenascin, in conjunction with a range of matrix metalloproteinases (MMPs), particularly MMP2 and MMP9, and to increase the expression of integrin receptors mediating the interactions with ECM $[1,3,5]$. However, it must be underlined that the transition from an epithelial to a mesenchymal cell phenotype is not merely an "on/off" state but rather a highly dynamic process evolving gradually [6].

\section{Molecular Players and Intracellular Pathways Regulating the "EMT Machinery"}

Gene expression changes in EMT are orchestrated by a number of transcription factors actively engaged in embryogenesis, such as Snail (Snail1), Slug (Snail2), Twist1/2, and ZEB1/2. Their activation in response to growth factors, cytokines, and morphogens $[13,14]$ is an early event during EMT. Indeed, EMT can be induced by a number of extracellular signals, whose downstream transduction pathways extensively crosstalk with each other, share effector molecules, and converge on common endpoints [15].

Members of the transforming growth factor- (TGF-) $\beta$ family (in particular TGF- $\beta 1$ ) are the prototypical activators of EMT. TGF- $\beta 1$ binding to the TGF- $\beta$ type II receptor results in the activation of the Smad signaling pathway, which induces the expression of EMT transcription factors (especially Snail and ZEB family members), and cooperates with them in promoting gene reprogramming. However, TGF- $\beta 1$ can also act through Smad-independent intracellular pathways, by activating Rho GTPases, mitogen-activated protein kinase (MAPK), and phosphoinositide 3-kinase (PI3K) [16, 17]. TGF- $\beta 1$ may act upon local activation by integrin $\alpha \mathrm{v} \beta 6$, which can be expressed at high levels by epithelial cells during tissue repair. Specifically, integrin $\alpha \mathrm{v} \beta 6$ cleaves the latencyassociated peptide from the latent precursor of TGF- $\beta 1$, which is otherwise sequestered in the ECM as inactive form. This mechanism of action is potentially relevant to promoting EMT changes, since, once locally activated, TGF- $\beta 1$ exerts its effects only within the limits of the epithelial cells displaying this specific receptor [18]. Alternatively, EMT initiation and progression can be regulated by tyrosine kinase receptors (RTKs), involved in signal transduction of epidermal growth factor (EGF), fibroblast growth factor (FGF), insulin-like growth factor, hepatocyte growth factor (HGF), and plateletderived growth factor (PDFG) [3]. RTKs stimulation has been widely linked to the activation of several regulatory molecules of EMT [19-23]. Notably, MAPK and PI3K pathways seem to play a major role in mediating RTKs-induced EMT [3]. Amongst morphogenetic signals, Wnt, Notch, and Hedgehog (Hh) signaling are well-established EMT inducers [24]. The ability of Wnt signals to trigger EMT relies on either the inhibition of the glycogen synthase kinase 3 (GSK3)- $\beta$, which prevents the destabilizing phosphorylation of Snail, or the nuclear translocation of $\beta$-catenin, whose gene targeting includes ZEB1 and Twist [25-27]. Notch and Hh signaling leading to the activation of EMT transcription factors (in particular the members of the Snail family) occurs through the activation of the Notch intracellular domain and the Gli family transcription factors, respectively [28-30].

The EMT program is finely regulated at a posttranscriptional level, by specific microRNAs (miRNA), including miR1, miR-29b, miR-34, miR-200, and miR-203. These are small RNAs with about twenty nucleotides, regulating stability and translational activity of mRNAs. In particular, miRNAs act in double-negative feedback loops with several EMT transcription factors, wherein they repress the expression of each other, thus providing epithelial cells with an additional, finely tuned mechanism aimed at maintaining EMT. Furthermore, a direct effect of miRNAs has been shown on the expression of critical biomarkers, such as E-cadherin, vimentin, and fibronectin (e.g., miR-9, miR-138, and miR-17), as well as on several EMT-promoting ligands and their related signaling pathways (e.g., miR-200a for $\beta$-catenin, miR-204 for TGF$\beta R 2$, miR-15, miR-16 for FGF, miR-198 for HGF [31], and miR-181a for TGF- $\beta$ [32]). Recent data indicate that miR$181 \mathrm{a}$ acts as a downstream effector of the TGF- $\beta$ signaling in hepatocytes where it modulates the expression of a number of EMT-related genes, among which are E-cadherin and vimentin [32]. In cholangiocytes, miRNA-15a downregulates Cdc25a, a cell-cycle regulator with potent proliferative effects, a mechanism possibly involved in hepatic cystogenesis [33].

It is important to underline that phenotypic changes resulting in EMT can be triggered by disease mechanisms, such as inflammation, hypoxia, ECM remodeling, and autophagy. In fact, proinflammatory cytokines, such as tumor necrosis factor- $\alpha(\mathrm{TNF} \alpha)$ and interleukin- $1 \beta$, and hypoxia can activate EMT master genes, acting through nuclear factor- (NF-) $\kappa \mathrm{B}$ and hypoxia inducible factor $1 \alpha$, respectively [34].

The pathological remodeling of ECM also represents an additional mechanism of EMT progression [3]. In this regard, epithelial cells exposed to MMP-3 upregulate an alternative splice isoform of Racl, which then enhances the expression of Snail by stimulating the production of reactive oxygen species [35]. Snail-induced EMT can be also triggered by type I collagen, which binds to $\alpha 2 \beta 1$ integrin causing an integrin linked kinase-mediated increase in nuclear NF- $\kappa \mathrm{B}$ activity [36].

Recent evidence suggest that also autophagy may behave as a critical regulator of EMT. Autophagy suppression by downmodulation of the autophagy-related gene 5 leads to the intracellular accumulation of the selective autophagy substrate p62, which then inhibits Twistl protein degradation in both autophagosomes and proteasome, thereby decreasing E-cadherin expression and promoting cell motility $[37,38]$.

\section{EMT in Liver Development}

Acquisition of a mesenchymal phenotype endowed with migratory functions is a prerequisite of many morphogenetic processes. This concept is well established in renal biology, given the mesodermal origin of the tubular epithelium, while it is less defined in the liver, where, instead, the epithelial cells (hepatocytes and cholangiocytes) derive from the foregut 
endoderm, and the mesodermal contribution is restricted to the generation of the stromal cells, including hepatic stellate cells (HSC). Only scant evidence suggest an EMT role in liver development. Studies performed in the late 1990s showed that cultured hepatocytes from neonatal rat livers underwent EMT changes, represented by the loss of specific differentiation markers, gain of a migrating morphology, and replacement of typical hepatocyte cytokeratins by vimentin, a property further stimulated by EGF [39]. EMT features were then reported in both hepatocytes and progenitor cells isolated from rodent and human fetal livers [40, 41]. Studies in mice from Lemaigre's group addressed the hypothesis that EMT is critically involved in the early process by which endodermal cells that line the hepatic diverticulum migrate through the basement membrane to invade the septum transversum, where they give rise to the hepatoblasts in the liver bud [42]. This process is controlled by the hematopoietically expressed homeobox factor (Hex), which acts in concert with the transcription factor GATA-6; their downmodulation is essential for hepatoblast clustering after liver budding. Expression of the prospero-related homeobox 1 (Prox-1), likely stimulated by the T-box transcription factor 3 (Tbx3), is an additional mechanism critically involved in hepatoblast migration, which interplays with Hex (Hex-Prox1 axis) [42]. Starting from this hypothesis, coexpression of K18 and $\alpha$-SMA was found in most nonhematopoietic cells of human fetal livers at early gestational ages; furthermore, multipotent stem cells expressing EMT features along with the stem cell markers Oct4 and Nanog were isolated in the human liver bud [43]. Unlike the early ontogenetic steps, data supporting an involvement of EMT in the morphogenesis of intrahepatic bile duct epithelium are even less evident. Expression of the SRY-related HMG box transcription factor 9 (SOX9) is critical for differentiation to a biliary epithelial phenotype. SOX9 is early expressed in endodermal cells of the hepatic diverticulum, but it is then downregulated as these cells are invading the septum transversum. SOX9 is reexpressed in the hepatoblasts switching to the ductal plate cell phenotype, and it is then maintained by cholangiocytes during the progressive maturation of bile ducts. When SOX9 is defective, epithelial cells become hyperresponsive to TGF$\beta$ [44], thereby being susceptible to mesenchymal changes. Thus, it seems that cholangiocytes express an active program to suppress EMT.

\section{In Chronic Cholangiopathies, Mesenchymal Markers Are Expressed by Ductular Reaction}

Broadly speaking, whereas activation of an EMT program may play a physiological role in embryonic development [2], its actual impact in disease conditions evolving to scarring is quite controversial, particularly in the liver [45]. Cholangiopathies may provide important clues to clarify whether and how EMT may really contribute to liver fibrogenesis. Cholangiopathies are a heterogeneous group of genetic and acquired liver disorders primarily targeting the epithelial cell lining the bile ducts (cholangiocyte). Most cholangiopathies typically follow a chronic, progressive course, characterized by an excessive matrix deposition confined to the portal tract (portal fibrosis), starting from the closest peribiliary area, and ultimately leading to portal hypertension, often before the development of full-blown cirrhosis. In contrast with other chronic liver diseases, treatment of cholangiopathies is mainly symptomatic, reflecting the limited knowledge on their pathogenesis. Nowadays, liver transplantation remains the only curative opportunity, especially in children and young adults $[46,47]$.

Fibrogenesis is the main determinant of disease progression, as well as of the most severe clinical manifestations related to portal hypertension, in both chronic hepatocellular damage and cholangiopathies. Fibrogenesis is a consequence of the excessive and sustained activation of tissue repair mechanisms driven by the ductular reaction [1]. Ductular reaction is a dynamic, multicellular reparative system that includes mesenchymal and inflammatory cells accompanying the expansion of the epithelial cells lining the smallest ramifications of the biliary tree, in continuity with the canals of Hering, which is the niche where the hepatic progenitor cells (HPC) is thought to reside. Expansion of the HPC compartment is a compensatory mechanism of liver repair activated when proliferative ability of mature liver cells is compromised because of a severe liver damage [48]. HPC are small cells marked with the bipotential capability to differentiate towards both biliary and hepatocyte lineages [49]. In ductular reaction, HPC-derived epithelial cells are arranged into irregular, highly branched ductules devoid of lumen, generally extending into the liver parenchyma and along the margins of the portal tract. During this process, ductular cells express a "reactive" phenotype (ductular reactive cells, DRC) and acquire the ability to produce cytokines, chemokines, growth factors, and angiogenic factors and to express a rich repertoire of receptors typically displayed by ductal plate cells in the early stages of liver development [50]. Thanks to these phenotypic changes, DRC may establish intense paracrine communications with multiple stromal cell types, including myofibroblasts (MFs), inflammatory cells, and endothelial cells, which dictate the functional consequences of ductular reaction [1]. To set in motion this multicellular reparative complex, DRC acquire a high degree of cell plasticity. Therefore, DRC need first to reduce the strength of cell-cell and cell-matrix contacts and then to acquire motile functions, enabling them to move from the HPC niche towards the site of damage whereby, by interacting with other inflammatory and mesenchymal cell elements, they build up the ductular reaction. A mainstay of the migratory properties of DRC is their increased production of polysialic acid (PolySia) in the course of biliary damage. PolySia is a highly polar ECM structural component with a strong binding affinity to the neural cell adhesion molecule (NCAM), commonly expressed by DRC [51]. PolySia turns NCAM adhesive properties into antiadhesive due to the size of the multiple PolySia chains and their high hydrophilic content $[52,53]$. This process is an essential step to promote plasticity and migration of $\mathrm{NCAM}^{+}$cells in the generation of ductular reaction. 


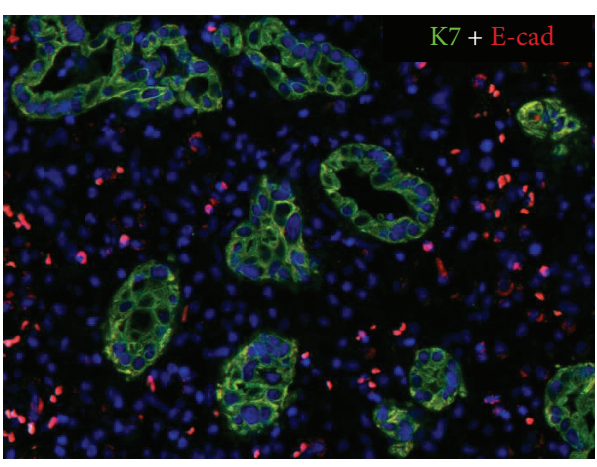

(a)

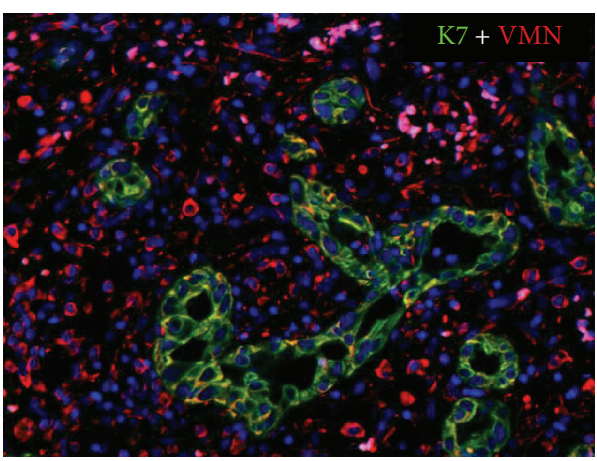

(c)

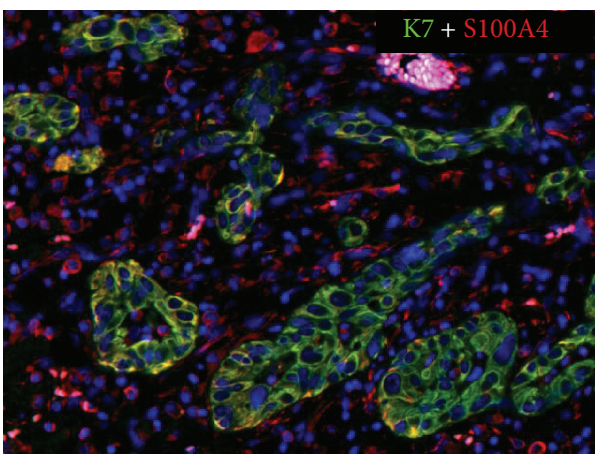

(e)

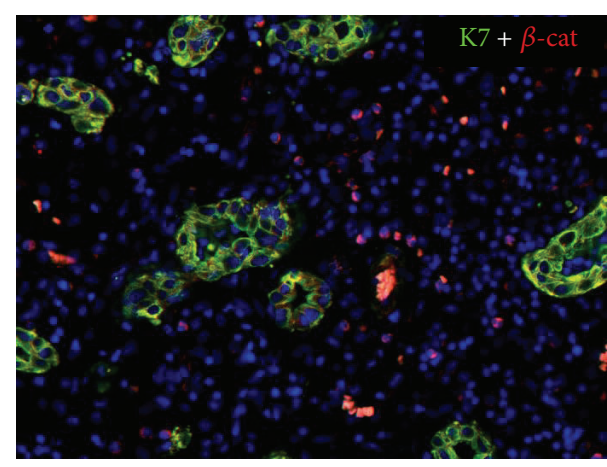

(b)

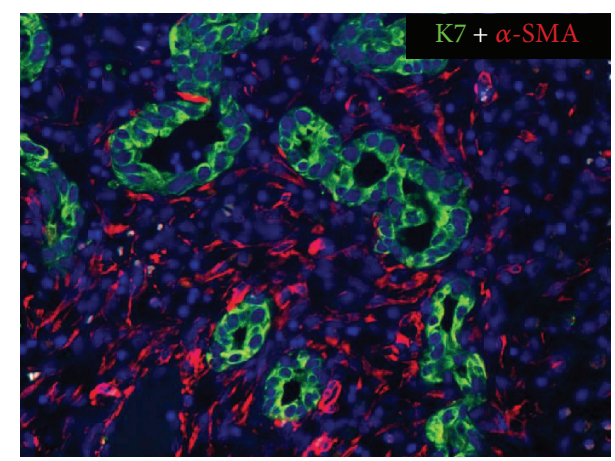

(d)

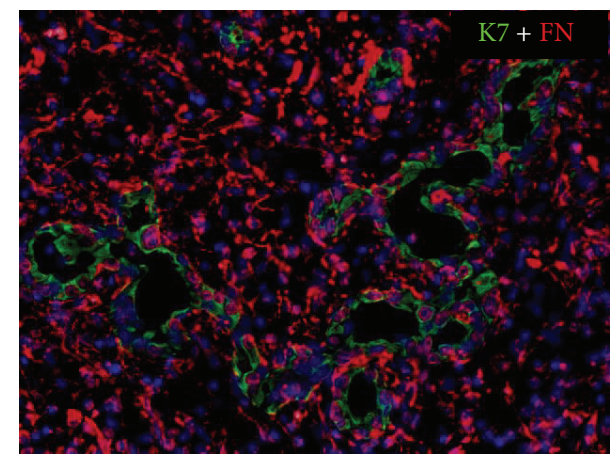

(f)

Figure 1: Partial expression of some mesenchymal features by ductular reactive cells. By dual immunofluorescence of a liver tissue section from a patient with ischemic cholangiopathy, with the cholangiocyte marker K7 (green), some mesenchymal features (red) are expressed by ductular reactive cells (coincident staining in yellow). They include downregulation of E-cadherin at the cell junctions of the epithelial layer (a) and upregulation in the cytoplasm of vimentin (c) and S100A4 (e) and in the basal side of fibronectin (f). In contrast, ductular reactive cells do not express typical markers of EMT, such as nuclear expression of $\beta$-catenin (b) and $\alpha$-SMA (d) $(\mathrm{M}=200 \mathrm{x})$.

Although heavily involved in fibrogenesis, DRC lack the ability to actively secrete ECM components, such as type I or type IV collagen, and must cooperate with other effector cells by stimulating their profibrotic activities. Among them, DRC interactions with portal MFs are a crucial step in fibrogenesis [54]. Portal MFs are fibrogenic cells localized within the portal space, characterized by spindle-shape morphology, $\alpha$-SMA expression, prominent motility, and contractility functions and strong capability to secrete ECM proteins, mostly type I collagen $[55,56]$. They may originate from multiple cell sources, including HSCs and, to a lesser extent, portal fibroblasts and bone marrow-derived mesenchymal stem cells. These are recruited by paracrine signals (TGF- $\beta$, PDGF-B, vascular endothelial growth factor, angiopoietin-1, and sphingosine 1-phosphate) released in the site of damage by the DRC as well as by the other components of the ductular reaction, such as macrophages and inflammatory cells $[1,57$, 58]. Whether the DRC themselves may be a further source of portal MFs via EMT as in the kidney $[59,60]$ and in the lung [61] has been hypothesized but never proven. However, several studies show that DRC express mesenchymal markers [62-64], as illustrated in Figure 1.

Initial studies showed that, in several cholangiopathies, cholangiocytes lining the small interlobular bile ducts and 
the reactive ductules lose some epithelial markers and acquire, in turn, several mesenchymal traits. Neoexpression of S100A4, vimentin, Snail, and MMP-2, associated with downregulation of E-cadherin and K19 in the bile ducts, were observed in histological samples of patients with primary biliary cirrhosis (PBC), primary sclerosing cholangitis [65], and biliary atresia (BA) $[62,66]$. Reduced expression of Ecadherin and increased expression of vimentin and S100A4 were also reported in bile ducts of patients with intrahepatic lithiasis, where these phenotypic changes strongly correlated with the extension of biliary fibrosis [67]. Epithelial expression of mesenchymal markers has been reported also in animal models of biliary fibrosis induced by bile duct ligation (BDL). In the BDL rat, cholangiocytes upregulate the expression of S100A4 and downregulate the expression of the specific epithelial markers and the membrane channel aquaporin-1, together with the cytokeratins K7 and K19 [7]. Similarly, in the BDL mice, small clusters of cholangiocytes showed immunoreactivity for both $\alpha$-SMA and heat-shock protein 47, a surrogate marker of type I collagen production, as well as migratory aspects into the periductal region [10]. However, it must be underlined that reliability of S100A4 as marker of EMT is limited by its concurrent expression by many inflammatory cells, including macrophages, often infiltrating the bile duct profile, thus posing a risk of misinterpreting histological sections.

Paracrine Hh signaling has been proposed by Omenetti and colleagues as a major driver of the EMT changes associated with biliary fibrosis in both rodents and humans [7]. In PBC, where EMT was originally proposed also as a mechanism contributing to ductopenia [68], Gli2, a transcription factor activated by Hh ligands, decorated the nuclei of ductular cells coexpressing both mesenchymal (S100A4, vimentin) and epithelial markers (K7) [7]. In BDL rats, relief of ductal obstruction reduced Hh pathway activity, an effect accompanied by reduction in EMT phenotype and biliary fibrosis [7]. Interestingly, mouse cholangiocytes cocultured with MFs, a rich source of soluble Hh ligands, acquired increased expression of several mesenchymal markers, including a migratory phenotype, while concomitantly repressing epithelial markers, and these effects were abolished by $\mathrm{Hh}$ antagonism [7]. Furthermore, EMT changes induced by $\mathrm{BDL}$ were exacerbated in transgenic mice harboring an overactivation of the Hh pathway caused by a defect in the Hh inhibitor Ptc [7]. The interplay between Hh activation and EMT was also reported in BA, a cholangiopathy featuring a pronounced ductular reaction associated with a rapid development of biliary fibrosis. In BA, a marked activation of the Hh signaling was associated with an excessive accumulation of ductular cells displaying an immature, mesenchymallike phenotype responsive to $\mathrm{Hh}$ [69]. Hh ligands could be also secreted by hepatocytes both in human liver diseases and in mice models of liver damage. Hh ligands, Sonic and Indian $\mathrm{Hh}$, both were overexpressed by hepatocytes in nonalcoholic steatohepatitis [70] and in chronic cholangiopathies such as primary biliary cirrhosis, respectively [71], in keeping with findings supporting a proapoptotic effect of Hh signaling [72]. Similarly, chronically liver injured mice by thioacetamide treatment showed an increased hepatocyte expression of Sonic and Indian $\mathrm{Hh}$ [73].

Noteworthy, Hh signaling may potentiate the pro-EMT effects of TGF- $\beta 1$, by interacting with its downstream effectors at several levels (Smad3, Snail, and Twist), and, in turn, liver cell expression of $\mathrm{Hh}$ ligands can be stimulated by TGF$\beta 1$ [74]. The crosstalk between the two signaling pathways is particularly relevant in biliary fibrosis, since TGF- $\beta 1$ is strongly upregulated in several cell types populating the ductular reaction, such as HSCs, endothelial cells, and Kupffer cells [1]. Given the effects on HPC and cholangiocytes, TGF- $\beta 1$ seems to play a pivotal role in the generation of ductular reaction. Within the HPC niche, some cells display mesenchymal-like features, which are modulated by TGF$\beta 1$ [75]. At high doses, TGF- $\beta 1$ is toxic to most epithelial cells, but, at low doses $(1-10 \mathrm{ng} / \mathrm{mL})$, it stimulates cultured cholangiocytes to acquire some mesenchymal markers, such as S100A4, vimentin, and $\alpha$-SMA, to lose expression of $\mathrm{K} 7$, $\mathrm{K} 19$, and E-cadherin and to gain invading abilities through the basement membrane $[10,65,66]$.

There is evidence suggesting that TGF- $\beta$ may drive expression of mesenchymal markers in cholangiocytes also in vivo in specific disease settings. For example, cholangiocytes display some mesenchymal features in both rat [76] and mouse (personal data) models of congenital hepatic fibrosis (CHF), a genetic cholangiopathy caused by mutations of the ciliary protein fibrocystin. CHF is characterized by progressive peribiliary fibrosis, accompanied by biliary dysgenesia. In the CHF mouse model, fibrocystin-defective cholangiocytes possess increased migratory functions [9], upregulate integrin $\alpha \mathrm{v} \beta 6$, and respond to TGF- $\beta 1$, by producing collagen type I. This feature is not observed in cultured normal cholangiocytes and may contribute to matrix deposition in the adjacent peribiliary area, where fibrogenesis starts (personal data). These phenotypic changes appear to be dependent upon an activation of the $\beta$-catenin signaling caused by a noncanonical phosphorylation at $\operatorname{Ser}^{675}$. Ser ${ }^{675}$ phosphorylation prevents $\beta$-catenin from degradation, thereby allowing its nuclear translocation and, subsequently, its transcriptional activity. Activation of $\beta$-catenin as observed in fibrocystindefective cholangiocytes is paradigmatic of the intracellular signaling perturbations induced by the loss of the tubular architecture and, consequently, of the cell polarity, which results in an increased secretion of cytokines, chemokines, and growth factors (personal data). This condition is likely reproduced by DRC, which, unlike normal cholangiocytes, are equipped with a number of ligand/receptor systems shared with the other cell elements involved in liver repair, mediating an extensive crosstalk ultimately leading to portal fibrogenesis [1].

Even in the model of fibrocystin deficiency, mesenchymal-like changes are induced in cholangiocytes by TGF$\beta 1$ released in the peribiliary space by progressively infiltrating macrophages, whose recruitment is regulated by a range of chemokines (CXCL1, CXC10, and CXCL12) secreted by cholangiocytes in a $\beta$-catenin-dependent fashion. Importantly, this signaling perturbation is specific to fibrocystin deficiency, as it is not observed in polycystic liver diseases 


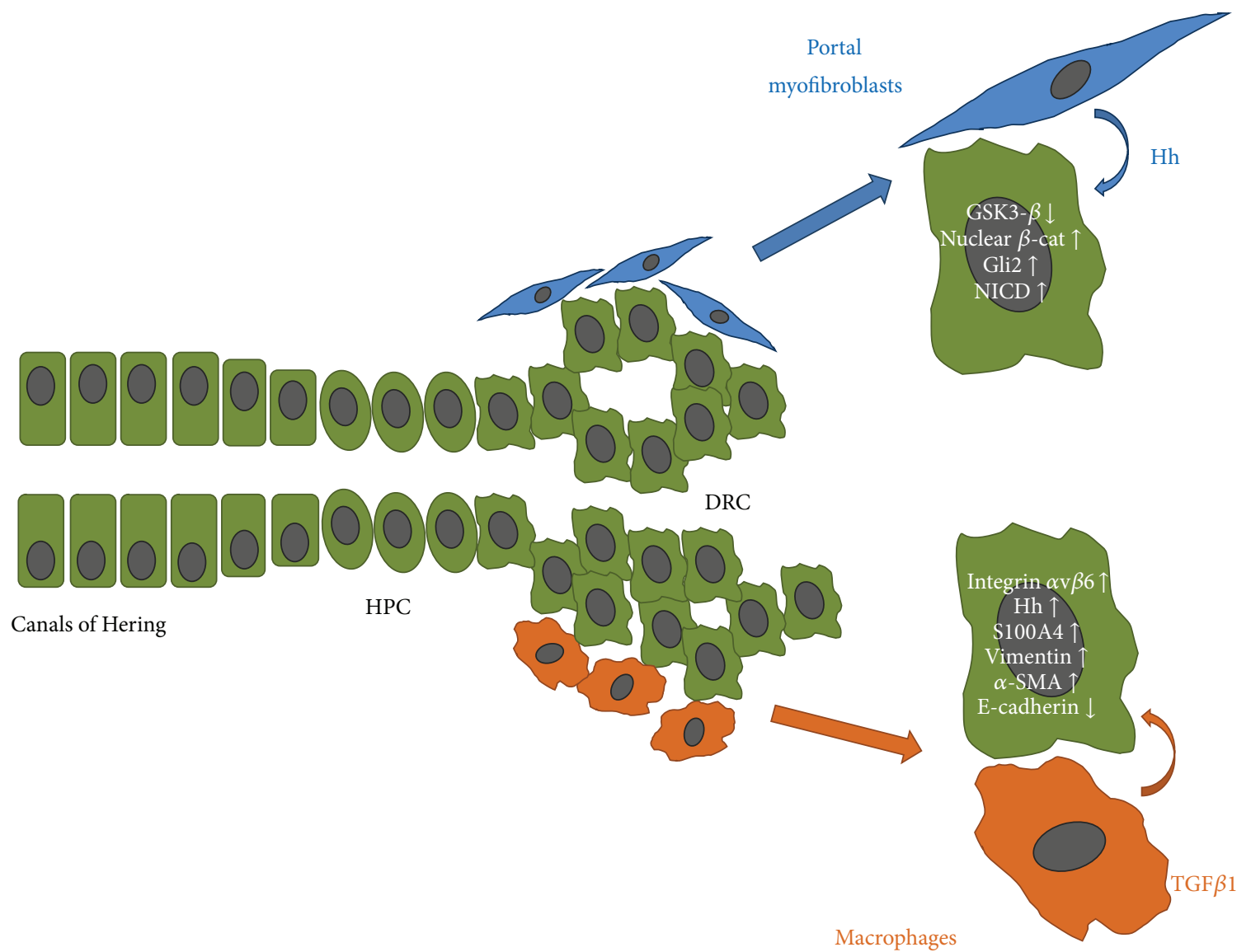

FIGURE 2: Epithelial-mesenchymal cell interactions promote ductular reaction. Crosstalk mechanisms with portal myofibroblasts and macrophages mediated by Hedgehog (Hh) and TGF- $\beta 1$, respectively, are critical in generating ductular reactive cells (DRC) from activation of the hepatic progenitor cell (HPC) compartment, residing in the niche nearby the canals of Hering. Hh and TGF- $\beta 1$ stimulate DRC to gain a range of mesenchymal changes typical of a "reactive" phenotype.

related to different ciliary protein defects, affecting polycystins [9]. However, even in this case, a full transdifferentiation of cholangiocytes into an activated mesenchymal phenotype ( $\alpha$-SMA neoexpression) was not found [76]. Crosstalk mechanisms driven by $\mathrm{Hh}$ ligands and TGF- $\beta 1$ released in the inflammatory microenvironment, promoting mesenchymal changes in DRC, are outlined in Figure 2. Collectively, the pro-EMT body of evidence in cholangiopathies is summarized in Table 1.

\section{Evidence against EMT in Biliary Fibrosis}

Most studies supporting the occurrence of EMT in cholangiocytes are based essentially on a morphological approach, even when taking advantage of elegant in vitro methodologies and well-characterized animal models. These findings were not confirmed in vivo by lineage-tracing experiments. In these studies, mice harboring a Cre recombinase under a cholangiocyte- or oval cell-specific promoter were crossed with a reporter strain carrying the yellow fluorescence protein (YFP) reporter gene preceded a floxed Stop cassette, and the progeny was then subjected to a cholestatic, fibrogenetic liver injury, caused by BDL.

In the first study [11], using K19-CreERT $\times$ Rosa26-YFP mice, immunostaining revealed that after experimental liver injury, mesenchymal markers such as $\alpha$-SMA, desmin (HSC biomarker), and S100A4 failed to colocalize in cholangiocytes tagged for K19 expression (i.e., $\mathrm{YFP}^{+}$cells), although, within the portal tract, $\mathrm{S} 100 \mathrm{~A}^{+}$and $\mathrm{K}^{+} 9^{+}$cells localized in close proximity to each other. These data indicate that, in vivo, cholangiocytes do not activate an EMT program. These results were confirmed in MFs isolated from BDL livers, where no $\mathrm{YFP}^{+}$cells could be detected. Similarly, in S100A4-green fluorescence protein (GFP) mice undergoing biliary damage, Pan- $\mathrm{K}^{+}$cholangiocytes never overlapped with S100A4-GFP ${ }^{+}$cells, thus confirming that cholangiocytes do not express S100A4. Even in this case, S100A4 ${ }^{+}$cells purified from cholestatic livers did not express panK, thus suggesting that a mesenchymal conversion of cholangiocytes, also transient, does not occur during liver injury. The discrepancy with Omenetti et al.s paper [7] may be related to the different biliary marker (K7 versus K19) used for coexpression studies with S100A4 [11]. In fact, the cholangiocyte 
TABLE 1: Summary of evidence in favor of or against the existence of EMT in biliary diseases.

\begin{tabular}{|c|c|c|}
\hline Model & Readouts & References \\
\hline \multicolumn{3}{|c|}{ Pro-EMT } \\
\hline Coculture of MFs and cholangiocytes & $\begin{array}{l}\text { Cholangiocytes: } \uparrow \text { S100A4, } \uparrow \text { Fibronectin, } \uparrow \mathrm{N} \text {-cadherin, and } \\
\text { increased motility }\end{array}$ & {$[7]$} \\
\hline $\begin{array}{l}\text { Cultured cholangiocytes from } \alpha \text {-fetoprotein (Alfp)-Cre } \times \\
\text { Rosa26-YFP mice treated with TGF } \beta \text {, or TNF } \alpha\end{array}$ & $\begin{array}{l}\uparrow \alpha \text {-SMA, loss of cell-cell contacts, cellular reshaping, and } \\
\text { E-cadherin delocalization }\end{array}$ & {$[8]$} \\
\hline Cultured cholangiocytes from $P k h d l^{d e l 4 / d e l 4}$ mouse & $\uparrow$ motility due to $\beta$-catenin activation & [9] \\
\hline BDL rat & Coexpression of S100A4 and vimentin with K7 & [7] \\
\hline BDL rat & $\begin{array}{l}\text { DRC (immunohistochemistry): } \uparrow \text { S100A4, } \uparrow \text { heat-shock protein } \\
47, \uparrow \alpha \text {-SMA } \downarrow \text { K7, } \downarrow \text { K19, and } \downarrow \text { Aquaporin- } 1\end{array}$ & [10] \\
\hline \multicolumn{3}{|c|}{ Against-EMT } \\
\hline K19-CreERT $\times$ Rosa26-YFP mice, BDL & No coexpression of K19 YFP with $\alpha$-SMA, Desmin, or S100A4 & [11] \\
\hline S100A4-CreERT $\times$ Rosa26-YFP mice, BDL & No coexpression of S100A4-GFP with Pan-K cells & [11] \\
\hline$\alpha$-fetoprotein (Alfp)-Cre $\times$ Rosa26-YFP mice, BDL & $\begin{array}{l}\text { No coexpression of YFP with S100A4, vimentin, } \alpha \text {-SMA, } \\
\text { procollagen } 1 \alpha 2 \text {, or desmin }\end{array}$ & {$[8]$} \\
\hline$\alpha$-fetoprotein (Alfp)-Cre $\times$ Rosa26-YFP mice, DDC & $\begin{array}{l}\text { No coexpression of YFP with S100A4, vimentin, } \alpha \text {-SMA, } \\
\text { procollagen } 1 \alpha 2 \text {, or desmin }\end{array}$ & {$[8]$} \\
\hline Human EGI-1-EGFP xenograft in SCID mice & $\begin{array}{l}\text { No K19/ } \alpha \text {-SMA coexpression; no expression of Y human } \\
\text { chromosome on } \alpha \text {-SMA }{ }^{+} \text {cells }\end{array}$ & {$[12]$} \\
\hline
\end{tabular}

population is highly heterogeneous and a distinct subset

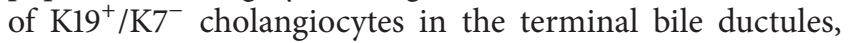
activated in specific disease conditions, has been identified [77]. On the contrary, a different subpopulation of $\mathrm{K}^{+} /{\mathrm{K} 19^{-}}^{-}$ cholangiocytes close to the HPC niche and mainly triggered by biliary damage has been also reported [78].

Therefore, to elude the technical trick related to the $\mathrm{K} 19^{+}$ cell fate mapping, in a second study, Chu and colleagues used the alpha-fetoprotein (Alfp)-Cre $\times$ Rosa26-YFP mouse [8]. Taking this approach, the authors could track the cell fate not only of $\mathrm{K}^{+} 9^{+}$cholangiocytes, but also of HPC. Again, no evidence of YFP colocalization with the mesenchymal markers S100A4, vimentin, $\alpha$-SMA, procollagen $1 \alpha 2$, or desmin was observed in liver tissue following BDL. Furthermore, no coexpression of the same markers by $\mathrm{YFP}^{+}$cells could be observed after 3,5-diethoxycarbonyl-1,4-dihydrocollidine (DDC) diet, a model of biliary damage known to generate a robust HPC activation. Overall, these data support the finding that neither cholangiocytes nor their cell progeny may convert into fibrogenic MFs during experimental cholestatic liver injury. However, in contrast with the in vivo data, in the same study, cultured cholangiocytes isolated from the reporter mice and challenged with TGF- $\beta 1$, alone or in combination with TNF- $\alpha$, showed loss of cell-cell contacts along with a fibroblastoid cell reshaping, intracellular delocalization of E-cadherin, and increased expression of $\alpha$-SMA. These apparent conflicting data clearly indicate that, under certain circumstances, cultured cholangiocytes may be committed to a complete EMT, unlike what happens in the in vivo condition, where the mesenchymal phenotype does not fully develop. This observation is in line with a recent study performed by our group to see if EMT contributes to the generation of the cancer-associated fibroblasts (CAF), usually accompanying the invasive growth of cholangiocarcinoma (CCA), a devastating malignancy originating from the biliary epithelium. A highly invasive human male CCA cell line (EGI-1) expressing an EMT phenotype, was xenografted by intraportal injection into a SCID male mouse, after transduction with lentiviral vectors encoding enhanced green fluorescence protein (EGFP). Liver tumors were analyzed by dual immunofluorescence for EGFP (serving as a CCA cell lineage marker) and $\alpha$-SMA (CAF marker). Indeed, engrafted tumors were closely surrounded by abundant CAF, thus reproducing the native CCA characteristics. In this model, cancer cells that underwent a complete EMT would be expected to coexpress both markers. However, coincident labeling between EGFP and $\alpha$-SMA was never observed in tissue samples from our xenograft models. Accordingly, FISH analysis further showed that the $\alpha-\mathrm{SMA}^{+}$cells expressed the murine rather than the human $\mathrm{Y}$ chromosome, which, instead, was normally expressed by infiltrating CCA cells [12].

Taken together, results of these fate-mapping studies are in accordance with a previous report showing the lack of EMT even in hepatocytes in a model of chronic hepatocellular damage [79] (Table 1). However, it must be underlined that experimental models of DDC and BDL are not fully consistent with the clinical phenotype of the human disease, most likely because of the rapid establishment of biliary fibrosis, which in chronic cholangiopathies takes instead several years to become clinically overt $[45,80,81]$. From this point of view, the CHF mouse model better reproduces the slow evolving tissue scarring seen in most human cholangiopathies.

\section{Conclusions}

The controversy on the role of EMT in biliary fibrosis is substantially a matter of definitions [1]. The term EMT should be abandoned in cholangiocyte biology. Nevertheless, cholangiocytes may acquire, to a variable degree, some mesenchymal properties as part of a "reactive" phenotype, 
which develops without the concurrent loss of the native epithelial identity. As pointed out by Kriz and colleagues, the main caveat of several studies favoring the EMT hypothesis is the a priori assumption of EMT as an established fact, leading to misinterpretation of data that are compatible with but not evidence for EMT [82]. Therefore, the risk is to overlook the high complexity of a process whose relevance is recognized only in renal biology.

In the setting of a reactive phenotype (ductular reaction and biliary dysgenesis), ECM components are abnormally represented in close vicinity of bile ducts. This feature is typical of several chronic cholangiopathies and represents the mechanism leading to portal hypertension and its severe complications. Therefore, it is expected that hampering fibrosis progression would lead to a significant improvement of patient's survival. However, the availability of effective antifibrotic therapies is still remote [83], especially for primary cholangiopathies [47]. The identification of factors released in the inflammatory microenvironment and able to activate DRC as well as of signaling perturbations modulating mesenchymal changes may provide a wide range of putative novel targets (soluble factors, morphogens, transcription factors, and miRNA) whose therapeutic interference might halt the progression of biliary fibrosis, an issue worth being investigated by future studies.

\section{Conflict of Interests}

The authors declare that there is no conflict of interests regarding the publication of this paper.

\section{Acknowledgments}

The authors acknowledge Fondazione Cariplo, Grant no. 2014-1099, to Massimiliano Cadamuro, and Telethon, Grant no. GGP09189, to Luca Fabris.

\section{References}

[1] L. Fabris and M. Strazzabosco, "Epithelial-mesenchymal interactions in biliary diseases," Seminars in Liver Disease, vol. 31, no. 1, pp. 11-32, 2011.

[2] J. P. Thiery, H. Acloque, R. Y. J. Huang, and M. A. Nieto, "Epithelial-mesenchymal transitions in development and disease," Cell, vol. 139, no. 5, pp. 871-890, 2009.

[3] S. Lamouille, J. Xu, and R. Derynck, "Molecular mechanisms of epithelial-mesenchymal transition," Nature Reviews Molecular Cell Biology, vol. 15, no. 3, pp. 178-196, 2014.

[4] R. Y.-J. Huang, P. Guilford, and J. P. Thiery, "Early events in cell adhesion and polarity during epithelial-mesenchymal transition," Journal of Cell Science, vol. 125, no. 19, pp. 4417-4422, 2012.

[5] J. J. Christiansen and A. K. Rajasekaran, "Reassessing epithelial to mesenchymal transition as a prerequisite for carcinoma invasion and metastasis," Cancer Research, vol. 66, no. 17, pp. 8319-8326, 2006.

[6] R. Kalluri and R. A. Weinberg, "The basics of epithelialmesenchymal transition," The Journal of Clinical Investigation, vol. 119, no. 6, pp. 1420-1428, 2009.
[7] A. Omenetti, A. Porrello, Y. Jung et al., "Hedgehog signaling regulates epithelial-mesenchymal transition during biliary fibrosis in rodents and humans," Journal of Clinical Investigation, vol. 118, no. 10, pp. 3331-3342, 2008.

[8] A. S. Chu, R. Diaz, J.-J. Hui et al., "Lineage tracing demonstrates no evidence of cholangiocyte epithelial-to-mesenchymal transition in murine models of hepatic fibrosis," Hepatology, vol. 53, no. 5, pp. 1685-1695, 2011.

[9] C. Spirli, L. Locatelli, C. M. Morell et al., "Protein kinase a-dependent $\mathrm{pSer}^{675}$ - $\beta$-catenin, a novel signaling defect in a mouse model of congenital hepatic fibrosis," Hepatology, vol. 58, no. 5, pp. 1713-1723, 2013.

[10] J.-L. Xia, C. Dai, G. K. Michalopoulos, and Y. Liu, "Hepatocyte growth factor attenuates liver fibrosis induced by bile duct ligation," American Journal of Pathology, vol. 168, no. 5, pp. 1500-1512, 2006.

[11] D. Scholten, C. H. Österreicher, A. Scholten et al., "Genetic labeling does not detect epithelial-to-mesenchymal transition of cholangiocytes in liver fibrosis in mice," Gastroenterology, vol. 139, no. 3, pp. 987-998, 2010.

[12] M. Cadamuro, G. Nardo, S. Indraccolo et al., "Platelet-derived growth factor-D and Rho GTPases regulate recruitment of cancer-associated fibroblasts in cholangiocarcinoma," Hepatology, vol. 58, no. 3, pp. 1042-1053, 2013.

[13] D. Hanahan and R. A. Weinberg, "Hallmarks of cancer: the next generation," Cell, vol. 144, no. 5, pp. 646-674, 2011.

[14] B. D. Craene and G. Berx, "Regulatory networks defining EMT during cancer initiation and progression," Nature Reviews Cancer, vol. 13, no. 2, pp. 97-110, 2013.

[15] J. P. Thiery and J. P. Sleeman, "Complex networks orchestrate epithelial-mesenchymal transitions," Nature Reviews Molecular Cell Biology, vol. 7, no. 2, pp. 131-142, 2006.

[16] Y. Katsuno, S. Lamouille, and R. Derynck, "TGF- $\beta$ signaling and epithelial-mesenchymal transition in cancer progression," Current Opinion in Oncology, vol. 25, no. 1, pp. 76-84, 2013.

[17] R. Derynck, B. P. Muthusamy, and K. Y. Saeteurn, "Signaling pathway cooperation in TGF- $\beta$-induced epithelialmesenchymal transition," Current Opinion in Cell Biology, vol. 31, pp. 56-66, 2014.

[18] E. Patsenker, Y. Popov, F. Stickel, A. Jonczyk, S. L. Goodman, and D. Schuppan, "Inhibition of integrin $\alpha \mathrm{v} \beta 6$ on cholangiocytes blocks transforming growth factor- $\beta$ activation and retards biliary fibrosis progression," Gastroenterology, vol. 135, no. 2, pp. 660-670, 2008.

[19] H.-W. Lo, S.-C. Hsu, W. Xia et al., "Epidermal growth factor receptor cooperates with signal transducer and activator of transcription 3 to induce epithelial-mesenchymal transition in cancer cells via up-regulation of TWIST gene expression," Cancer Research, vol. 67, no. 19, pp. 9066-9076, 2007.

[20] Z.-C. Liu, H.-S. Wang, G. Zhang et al., "AKT/GSK-3 $\beta$ regulates stability and transcription of snail which is crucial for bFGFinduced epithelial-mesenchymal transition of prostate cancer cells," Biochimica et Biophysica Acta: General Subjects, vol. 1840, no. 10, pp. 3096-3105, 2014.

[21] T. R. Graham, H. E. Zhau, V. A. Odero-Marah et al., "Insulinlike growth factor-I-dependent up-regulation of ZEB1 drives epithelial-to-mesenchymal transition in human prostate cancer cells," Cancer Research, vol. 68, no. 7, pp. 2479-2488, 2008.

[22] S. Grotegut, D. von Schweinitz, G. Christofori, and F. Lehembre, "Hepatocyte growth factor induces cell scattering through MAPK/Egr-1-mediated upregulation of Snail," The EMBO Journal, vol. 25, no. 15, pp. 3534-3545, 2006. 
[23] D. Kong, Y. Li, Z. Wang et al., "miR-200 regulates PDGFD-mediated epithelial-mesenchymal transition, adhesion, and invasion of prostate cancer cells," Stem Cells, vol. 27, no. 8, pp. 1712-1721, 2009.

[24] D. M. Gonzalez and D. Medici, "Signaling mechanisms of the epithelial-mesenchymal transition," Science Signaling, vol. 7, no. 344, article re8, 2014.

[25] B. P. Zhou, J. Deng, W. Xia et al., "Dual regulation of Snail by GSK-3 $\beta$-mediated phosphorylation in control of epithelialmesenchymal transition," Nature Cell Biology, vol. 6, no. 4, pp. 931-940, 2004.

[26] U. D. Kahlert, D. Maciaczyk, S. Doostkam et al., "Activation of canonical WNT/ $\beta$-catenin signaling enhances in vitro motility of glioblastoma cells by activation of ZEB1 and other activators of epithelial-to-mesenchymal transition," Cancer Letters, vol. 325, no. 1, pp. 42-53, 2012.

[27] L. R. Howe, O. Watanabe, J. Leonard, and A. M. C. Brown, "Twist is up-regulated in response to Wntl and inhibits mouse mammary cell differentiation," Cancer Research, vol. 63, no. 8, pp. 1906-1913, 2003.

[28] K. G. Leong, K. Niessen, I. Kulic et al., "Jagged1-mediated Notch activation induces epithelial-to-mesenchymal transition through Slug-induced repression of E-cadherin," Journal of Experimental Medicine, vol. 204, no. 12, pp. 2935-2948, 2007.

[29] S. Saad, S. R. Stanners, R. Yong, O. Tang, and C. A. Pollock, "Notch mediated epithelial to mesenchymal transformation is associated with increased expression of the Snail transcription factor," International Journal of Biochemistry and Cell Biology, vol. 42, no. 7, pp. 1115-1122, 2010.

[30] X. Li, W. Deng, C. D. Nail et al., "Snail induction is an early response to Glil that determines the efficiency of epithelial transformation," Oncogene, vol. 25, no. 4, pp. 609-621, 2006.

[31] F. Guo, B. C. Parker Kerrigan, D. Yang et al., "Post-transcriptional regulatory network of epithelial-to-mesenchymal and mesenchymal-to-epithelial transitions," Journal of Hematology and Oncology, vol. 7, article 19, 2014.

[32] J. Brockhausen, S. S. Tay, C. A. Grzelak et al., "miR-181a mediates TGF- $\beta$-induced hepatocyte EMT and is dysregulated in cirrhosis and hepatocellular cancer," Liver International, vol. 35, no. 1, pp. 240-253, 2015.

[33] S.-O. Lee, T. Masyuk, P. Splinter et al., "MicroRNA15a modulates expression of the cell-cycle regulator Cdc25A and affects hepatic cystogenesis in a rat model of polycystic kidney disease," Journal of Clinical Investigation, vol. 118, no. 11, pp. 3714-3724, 2008.

[34] S. Lindsey and S. A. Langhans, "Crosstalk of oncogenic signaling pathways during epithelial-mesenchymal transition," Frontiers in Oncology, vol. 4, article 358, 2014.

[35] D. C. Radisky, D. D. Levy, L. E. Littlepage et al., "Raclb and reactive oxygen species mediate MMP-3-induced EMT and genomic instability," Nature, vol. 436, no. 7047, pp. 123-127, 2005.

[36] D. Medici and A. Nawshad, "Type I collagen promotes epithelial-mesenchymal transition through ILK-dependent activation of NF- $\kappa$ B and LEF-1," Matrix Biology, vol. 29, no. 3, pp. 161-165, 2010.

[37] L. Qiang, B. Zhao, M. Ming et al., "Regulation of cell proliferation and migration by 62 through stabilization of Twist1," Proceedings of the National Academy of Sciences of the United States of America, vol. 111, no. 25, pp. 9241-9246, 2014.
[38] L. Qiang and Y.-Y. He, "Autophagy deficiency stabilizes TWIST1 to promote epithelial-mesenchymal-transition," Autophagy, vol. 10, no. 10, pp. 1864-1865, 2014.

[39] R. Pagan, M. Llobera, and S. Vilaró, "Epithelial-mesenchymal transition in cultured neonatal hepatocytes," Hepatology, vol. 21, no. 3, pp. 820-831, 1995.

[40] F. Valdés, A. M. Álvarez, A. Locascio et al., "The epithelial mesenchymal transition confers resistance to the apoptotic effects of transforming growth factor $\beta$ in fetal rat hepatocytes," Molecular Cancer Research, vol. 1, no. 1, pp. 68-78, 2002.

[41] Y. Y. Dan, K. J. Riehle, C. Lazaro et al., "Isolation of multipotent progenitor cells from human fetal liver capable of differentiating into liver and mesenchymal lineages," Proceedings of the National Academy of Sciences of the United States of America, vol. 103, no. 26, pp. 9912-9917, 2006.

[42] F. P. Lemaigre, "Mechanisms of liver development: concepts for understanding liver disorders and design of novel therapies," Gastroenterology, vol. 137, no. 1, pp. 62-79, 2009.

[43] J. Su, P. You, W.-L. Li et al., "The existence of multipotent stem cells with epithelial-mesenchymal transition features in the human liver bud," International Journal of Biochemistry and Cell Biology, vol. 42, no. 12, pp. 2047-2055, 2010.

[44] A. Antoniou, P. Raynaud, S. Cordi et al., "Intrahepatic bile ducts develop according to a new mode of tubulogenesis regulated by the transcription factor SOX9," Gastroenterology, vol. 136, no. 7, pp. 2325-2333, 2009.

[45] R. G. Wells, "The epithelial-to-mesenchymal transition in liver fibrosis: here today, gone tomorrow?" Hepatology, vol. 51, no. 3, pp. 737-740, 2010.

[46] K. N. Lazaridis, M. Strazzabosco, and N. F. Larusso, "The cholangiopathies: disorders of biliary epithelia," Gastroenterology, vol. 127, no. 5, pp. 1565-1577, 2004.

[47] M. Strazzabosco, L. Fabris, and C. Spirli, "Pathophysiology of cholangiopathies," Journal of Clinical Gastroenterology, vol. 39, no. 4, pp. S90-S102, 2005.

[48] T. Roskams, "Progenitor cell involvement in cirrhotic human liver diseases: from controversy to consensus," Journal of Hepatology, vol. 39, no. 3, pp. 431-434, 2003.

[49] T. A. Roskams, N. D. Theise, C. Balabaud et al., "Nomenclature of the finer branches of the biliary tree: canals, ductules, and ductular reactions in human livers," Hepatology, vol. 39, no. 6, pp. 1739-1745, 2004.

[50] M. Strazzabosco and L. Fabris, "Development of the bile ducts: essentials for the clinical hepatologist," Journal of Hepatology, vol. 56, no. 5, pp. 1159-1170, 2012.

[51] L. Fabris, M. Strazzabosco, H. A. Crosby et al., "Characterization and isolation of ductular cells coexpressing neural cell adhesion molecule and $\mathrm{Bcl}-2$ from primary cholangiopathies and ductal plate malformations," The American Journal of Pathology, vol. 156, no. 5, pp. 1599-1612, 2000.

[52] A. Tsuchiya, W. Y. Lu, B. Weinhold et al., "Polysialic acid/neural cell adhesion molecule modulates the formation of ductular reactions in liver injury," Hepatology, vol. 60, no. 5, pp. 17271740, 2014.

[53] M. Strazzabosco and L. Fabris, "Neural cell adhesion molecule and polysialic acid in ductular reaction: the puzzle is far from completed, but the picture is becoming more clear," Hepatology, vol. 60, no. 5, pp. 1469-1472, 2014.

[54] S. M. Park, "The crucial role of cholangiocytes in cholangiopathies," Gut and Liver, vol. 6, no. 3, pp. 295-304, 2012. 
[55] M. Parola, F. Marra, and M. Pinzani, "Myofibroblast-like cells and liver fibrogenesis: emerging concepts in a rapidly moving scenario," Molecular Aspects of Medicine, vol. 29, no. 1-2, pp. 5866, 2008.

[56] K. Iwaisako, D. A. Brenner, and T. Kisseleva, "What's new in liver fibrosis? The origin of myofibroblasts in liver fibrosis," Journal of Gastroenterology and Hepatology, vol. 27, supplement 2, pp. 6568, 2012.

[57] E. Novo, S. Cannito, E. Zamara et al., "Proangiogenic cytokines as hypoxia-dependent factors stimulating migration of human hepatic stellate cells," The American Journal of Pathology, vol. 170, no. 6, pp. 1942-1953, 2007.

[58] C. Li, Y. Kong, H. Wang et al., "Homing of bone marrow mesenchymal stem cells mediated by sphingosine 1-phosphate contributes to liver fibrosis," Journal of Hepatology, vol. 50, no. 6, pp. 1174-1183, 2009.

[59] M. Iwano, D. Plieth, T. M. Danoff, C. Xue, H. Okada, and E. G. Neilson, "Evidence that fibroblasts derive from epithelium during tissue fibrosis," Journal of Clinical Investigation, vol. 110, no. 3, pp. 341-350, 2002.

[60] R. Kalluri and E. G. Neilson, "Epithelial-mesenchymal transition and its implications for fibrosis," The Journal of Clinical Investigation, vol. 112, no. 12, pp. 1776-1784, 2003.

[61] K. K. Kim, M. C. Kugler, P. J. Wolters et al., "Alveolar epithelial cell mesenchymal transition develops in vivo during pulmonary fibrosis and is regulated by the extracellular matrix," Proceedings of the National Academy of Sciences of the United States of America, vol. 103, no. 35, pp. 13180-13185, 2006.

[62] R. Díaz, J. W. Kim, J. J. Hui et al., "Evidence for the epithelial to mesenchymal transition in biliary atresia fibrosis," Human Pathology, vol. 39, no. 1, pp. 102-115, 2008.

[63] Y.-H. Deng, C.-L. Pu, Y.-C. Li et al., "Analysis of biliary epithelial-mesenchymal transition in portal tract fibrogenesis in biliary atresia," Digestive Diseases and Sciences, vol. 56, no. 3, pp. 731-740, 2011.

[64] J. M. Lee, S. Dedhar, R. Kalluri, and E. W. Thompson, "The epithelial-mesenchymal transition: new insights in signaling, development, and disease," Journal of Cell Biology, vol. 172, no. 7, pp. 973-981, 2006.

[65] K. A. Rygiel, H. Robertson, H. L. Marshall et al., "Epithelialmesenchymal transition contributes to portal tract fibrogenesis during human chronic liver disease," Laboratory Investigation, vol. 88, no. 2, pp. 112-123, 2008.

[66] K. Harada, Y. Sato, H. Ikeda et al., "Epithelial-mesenchymal transition induced by biliary innate immunity contributes to the sclerosing cholangiopathy of biliary atresia," Journal of Pathology, vol. 217, no. 5, pp. 654-664, 2009.

[67] R. Sung, S. H. Lee, M. Ji et al., "Epithelial-mesenchymal transition-related protein expression in biliary epithelial cells associated with hepatolithiasis," Journal of Gastroenterology and Hepatology, vol. 29, no. 2, pp. 395-402, 2014.

[68] H. Robertson, J. A. Kirby, W. W. Yip, D. E. J. Jones, and A. D. Burt, "Biliary epithelial-mesenchymal transition in posttransplantation recurrence of primary biliary cirrhosis," Hepatology, vol. 45, no. 4, pp. 977-981, 2007.

[69] A. Omenetti, L. M. Bass, R. A. Anders et al., "Hedgehog activity, epithelial-mesenchymal transitions, and biliary dysmorphogenesis in biliary atresia," Hepatology, vol. 53, no. 4, pp. 12461258, 2011.

[70] W.-K. Syn, Y. Jung, A. Omenetti et al., "Hedgehog-mediated epithelial-to-mesenchymal transition and fibrogenic repair in nonalcoholic fatty liver disease," Gastroenterology, vol. 137, no. 4, pp. e1478-e1488, 2009.

[71] Y. Jung, S. J. McCall, Y.-X. Li, and A. M. Diehl, "Bile ductules and stromal cells express hedgehog ligands and/or hedgehog target genes in primary biliary cirrhosis," Hepatology, vol. 45, no. 5, pp. 1091-1096, 2007.

[72] Y. Jung, R. P. Witek, W.-K. Syn et al., "Signals from dying hepatocytes trigger growth of liver progenitors," Gut, vol. 59, no. 5, pp. 655-665, 2010.

[73] C. A. Grzelak, L. G. Martelotto, N. D. Sigglekow et al., "The intrahepatic signalling niche of hedgehog is defined by primary cilia positive cells during chronic liver injury," Journal of Hepatology, vol. 60, no. 1, pp. 143-151, 2014.

[74] Y. Jung, K. D. Brown, R. P. Witek et al., "Accumulation of hedgehog-responsive progenitors parallels alcoholic liver disease severity in mice and humans," Gastroenterology, vol. 134, no. 5, pp. 1532.e3-1543.e3, 2008.

[75] G. K. Michalopoulos, "Liver regeneration," Journal of Cellular Physiology, vol. 213, no. 2, pp. 286-300, 2007.

[76] Y. Sato, K. Harada, S. Ozaki et al., "Cholangiocytes with mesenchymal features contribute to progressive hepatic fibrosis of the polycystic kidney rat," American Journal of Pathology, vol. 171, no. 6, pp. 1859-1871, 2007.

[77] S. Paku, K. Dezso, L. Kopper, and P. Nagy, "Immunohistochemical analysis of cytokeratin 7 expression in resting and proliferating biliary structures of rat liver," Hepatology, vol. 42, no. 4, pp. 863-870, 2005.

[78] H. A. Crosby, S. Hubscher, L. Fabris et al., "Immunolocalization of putative human liver progenitor cells in livers from patients with end-stage primary biliary cirrhosis and sclerosing cholangitis using the monoclonal antibody OV-6," The American Journal of Pathology, vol. 152, no. 3, pp. 771-779, 1998.

[79] K. Taura, K. Miura, K. Iwaisako et al., "Hepatocytes do not undergo epithelial-mesenchymal transition in liver fibrosis in mice," Hepatology, vol. 51, no. 3, pp. 1027-1036, 2010.

[80] T. Kisseleva and D. A. Brenner, "Is it the end of the line for the EMT?” Hepatology, vol. 53, no. 5, pp. 1433-1435, 2011.

[81] S.-J. Lee, K.-H. Kim, and K.-K. Park, "Mechanisms of fibrogenesis in liver cirrhosis: the molecular aspects of epithelialmesenchymal transition," World Journal of Hepatology, vol. 6, no. 4, pp. 207-216, 2014.

[82] W. Kriz, B. Kaissling, and M. Le Hir, "Epithelial-mesenchymal transition (EMT) in kidney fibrosis: fact or fantasy?" Journal of Clinical Investigation, vol. 121, no. 2, pp. 468-474, 2011.

[83] A. Mallat and S. Lotersztajn, "Cellular mechanisms of tissue fibrosis. 5. novel insights into liver fibrosis," American Journal of Physiology-Cell Physiology, vol. 305, no. 8, pp. C789-C799, 2013. 

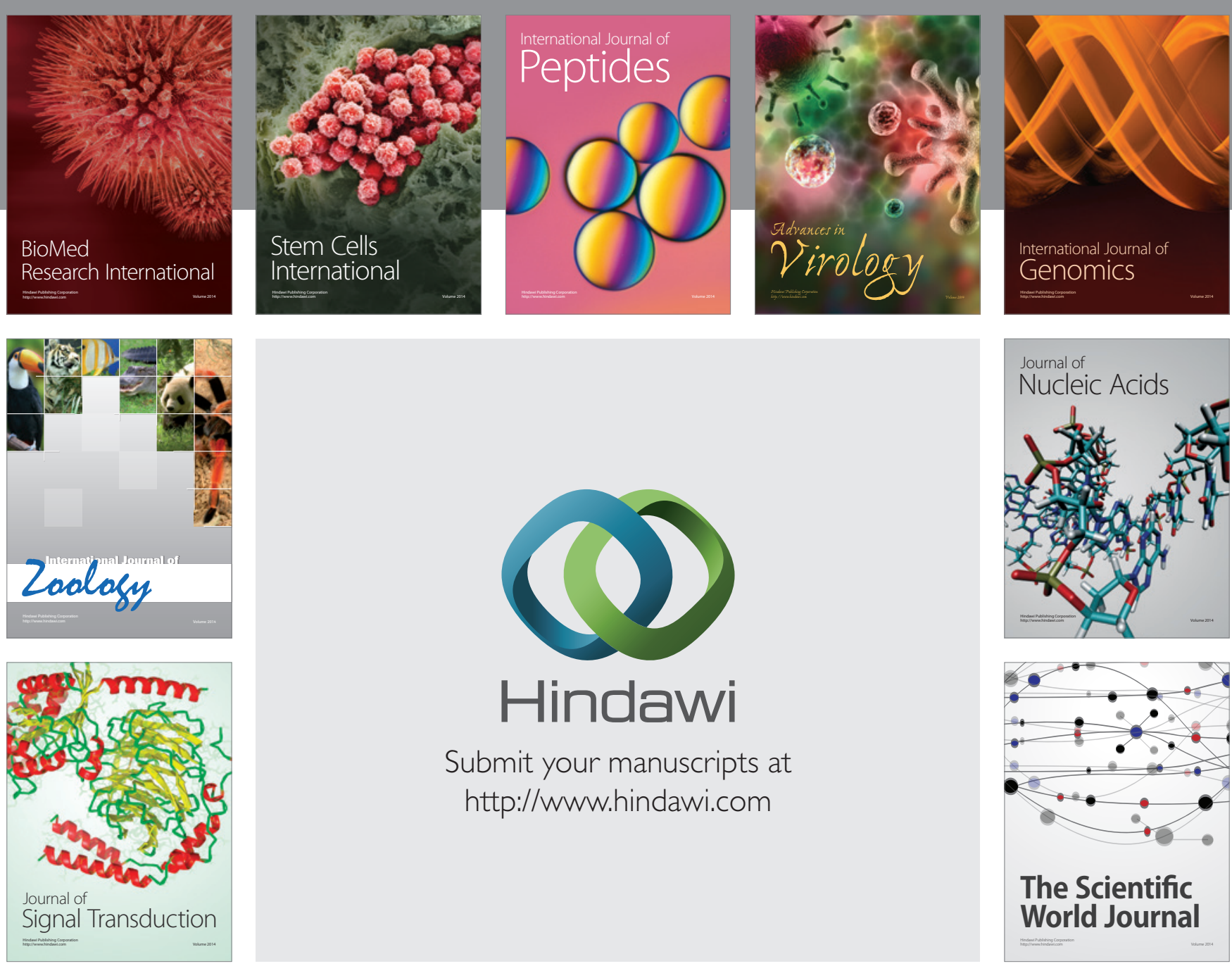

Submit your manuscripts at

http://www.hindawi.com
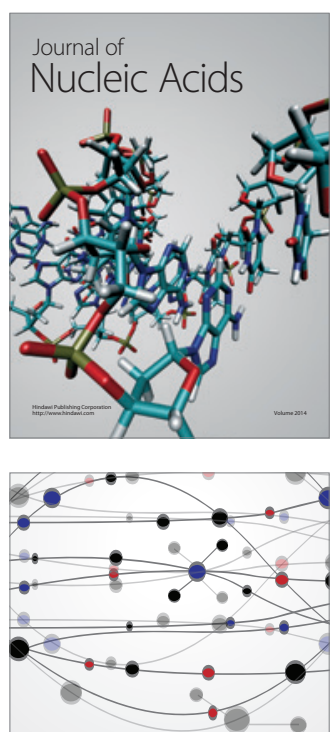

The Scientific World Journal
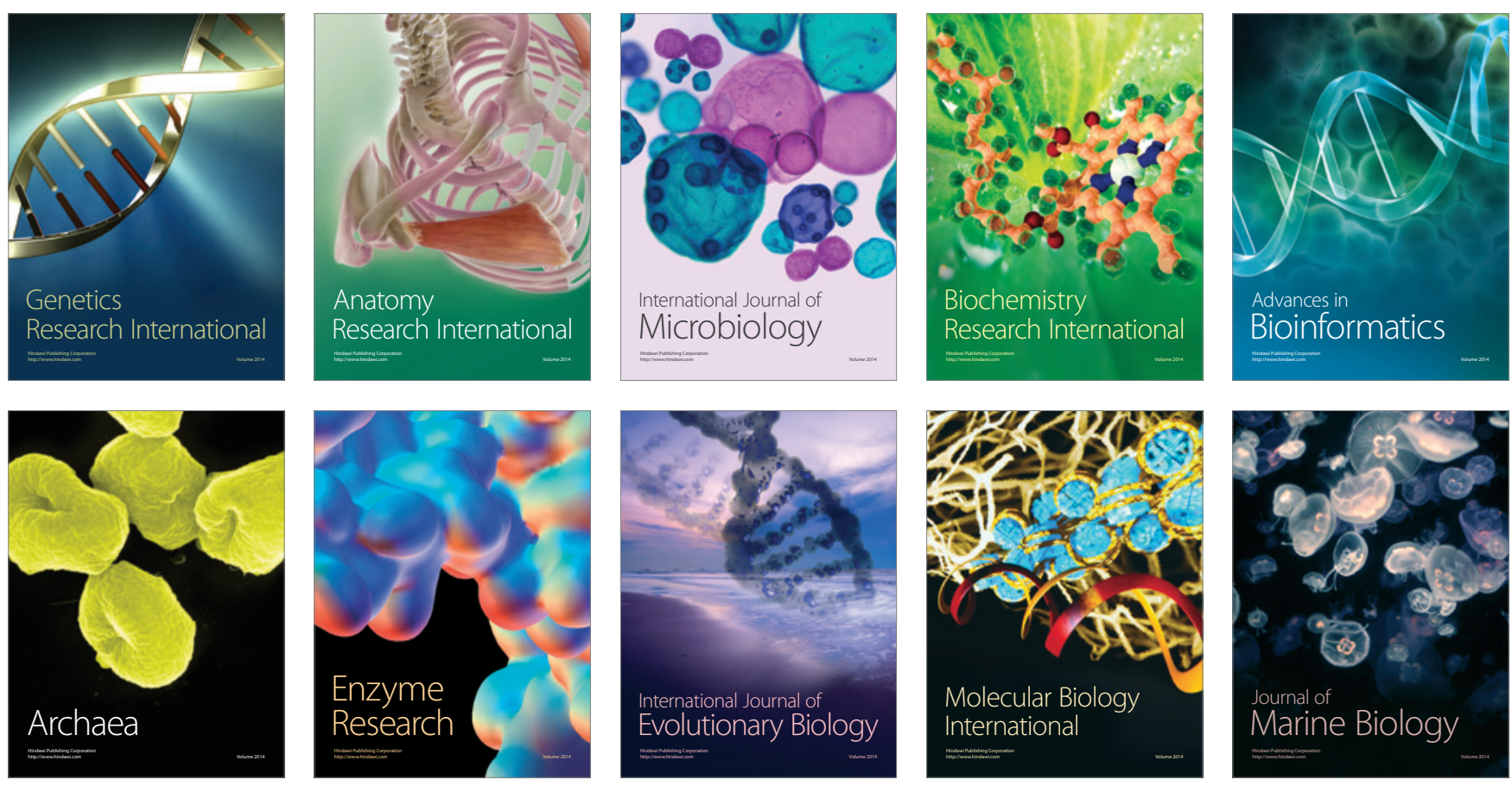